\title{
Single-tree influence of Tectona grandis Linn. f. on plant distribution and soil characteristics in a planted forest
}

\author{
Beckley Ikhajiagbe ${ }^{1}$, Matthew Chidozie Ogwu ${ }^{1,2^{*}}$ (iD and Adebayo Emmanuel Lawrence ${ }^{1,3}$
}

\begin{abstract}
Background: Little is known about the single-tree influence of Tectona grandis Linn. f. on plant distribution and soil characteristics in Benin City, Nigeria. We investigated the possible single-tree effect of T. grandis on understory plants in an 8-year-old teak plantation at the Moist Forest Research Station. An area of $36.57 \mathrm{~m}$ by $60.96 \mathrm{~m}$ was marked out and divided into 15 equal-sized subplots containing 10 trees per subplot. Marked distances from the base of a randomly selected tree per subplot were made (0-0.5 m, 0.5-1.0 m and 1.0-1.5 m).

Results: Single-tree influence of $T$. grandis was observed in the soil total organic carbon, total nitrogen and soluble phosphorus, where concentrations were higher with 1.5 -m radius from the tree than beyond. Moreover, the $\mathrm{pH}$ of the topsoil within $1.5 \mathrm{~m}$ from the base of the tree was lower $(\mathrm{pH} 4.4)$ than beyond $1.5 \mathrm{~m}$ from the base of the tree (pH 5.4). Species-specific single-tree effect was also observed on the understory plant distribution likely due to diverse ecophysiological interactions. Within $1.5 \mathrm{~m}$ from the tree, plant species abundance, especially of Sida garckeana, Reisantia indica, Momordica charantia and Tridax procumbens were negatively affected. However, the distribution of Eleusine indica around the tree was not negatively influenced. Plant abundance was generally suppressed in Cynodon dactylon, Axonopus compressus, Andropogon gayanus, Commelina diffusa and Euphorbia hirta. Generally, there were more plant species beyond the canopy fringes than within the canopy, indicating inhibitory single-tree effects.

Conclusion: Not all plant species in close proximity to T. grandis are affected. This is important considering that plant-plant associations affect the quality of forest soils. Generally, more plant species were recorded outside the 1.5-m demarcation than within, an increase in soil organic matter may further enhance such plant species abundance. The impact of $T$. grandis in forest soil quality is possibly a factor of the outcome of its association with neighbouring plant species. Diverse mechanisms at play may be responsible for the observed effects on soil chemistry. However, a reduction in the soil organic matter and variations in other environmental factors also contributed to observed single-tree effect.
\end{abstract}

Keywords: Plant diversity, Tropical forest, Single-tree effect, Tectona grandis Plantation, Understory, Forest weeds

\section{Background}

The importance of trees is underscored by their economic and environmental roles. Some tree populations have been known to control the overall plant community

\footnotetext{
* Correspondence: matthew.ogwu@uniben.edu

${ }^{1}$ Department of Plant Biology and Biotechnology, Faculty of Life Sciences University of Benin, Ugbowo, Benin City PMB 1154, Nigeria

${ }^{2} \mathrm{~S} c h o o l$ of Bioscience and Veterinary Medicine, University of Camerino Center for Floristic Research of the Apennine, Gran Sasso and Monti della Laga National Park, San Colombo, 67021 Barisciano, L'Aquila, Italy Full list of author information is available at the end of the article
}

likely because of their biomass (Thakur and Eisenhauer, 2015; Jia et al., 2018). By so doing, they create a microenvironment through the diminished air and soil temperatures and reduce wind speeds enabling the formation of smaller niches within the forest ecosystem. Jose et al. (2008); Rao et al. (1998) posited that these events culminate in ecophysiological changes such as decreased evaporation with enhanced humidity in forest areas. Moreover, the availability of water in the forest to plants, particularly those within close proximity to tree roots has been reported (Burgess et al., 1998; Ong et al., 
1999). Through the processes of hydraulic lift, these trees help to transport water from deep soil layers, which were hitherto impossible for most of the understory herbaceous plants, to drier surfaces, which were bedevilled with competition for water and other nutrients. In turn, plant abundance within tree canopies may be attributed to protection from extreme irradiation and heat effects, which invariably increases the rate of water loss by these plants (Lopez-Pintor et al. 2000). From the foregoing, it is evident that the understory regions of trees are possible microhabitats for these sets of plants.

Another consideration is many plant populations never exist in isolation of other plants. Rather, as outlined in Riginos (2009), their coexistence and interaction ultimately give rise to codominance. However, such associations may become hampered in the event that the associated tree exhibits some level of allelopathy, or the capacity to alter the availability of soil nutrients, light and other limiting resources. On the other hand, the growth performance of any tree may be affected by a number of factors including cultural practices, planting density, as well as the tree's interaction with understory plants that also significant from an ecological viewpoint (Cantarelli et al. 2006, Leopold and Salazar 2008, Silva et al. 2010).

The ability of natural forests to meet the demand for timber requirements was evidently in doubt in Nigeria; hence, the intensification in afforestation programmes in Nigeria has been performed by the Department of Forestry and the Federal Ministry of Environment. One of the tree species adopted for afforestation programme was teak (Tectona grandis Linn. f. Verbenaceae). Actually, the usefulness and popularity of teak have been known for many centuries, which contributed to the relatively widespread distribution and cultivation throughout the tropics. Generally, the agroforestry deliberately combines tree cultivation with crops and pasture production. Accordingly, the success of the agroforestry system depends on the choice of both tree and associated crop or pasture. In either case, guaranteeing the total development of the tree is paramount; hence, the distribution of the understory plants cannot be overlooked. The question, therefore, is whether the singletree influence on the distribution of the associated weeds counts one way or the other.

Inderjit and Callaway (2003) reported that it is important to study spatial patterns of the weeds in the field as it relates to silviculture as well as growth inhibition zones as this point to the allelopathic disposition of the trees in question. Consequently, the deliberate investigation of plant species beneath the canopy of the tree would help to pinpoint possible beneficial plant species that may be useful in weed control via allelopathy. In a bid to guarantee sustainable practice in agricultural development, allelochemicals are being viewed as possible alternatives to synthetic agrochemicals (Scrivanti et al. 2003, Maraschin-Silva and Aqüila 2006). Some of these allelochemicals, otherwise known as functional allelochemicals (Aldrich, 1984) are transformed by soil microorganisms, and as such has influence in the activity and distribution of soil microorganisms.

The capacity, however, for $T$. grandis to exhibit a negative influence on plant development has been previously reported (Kole et al. 2011, Manimegalai 2013). Kole et al. (2011) investigated allelopathic effects of teak leaf extract on junglerice (Echinochloa colona) and sedge (Cyperus difformis) in a rice farm. They reported no significant effects on rice germination, but inhibitory activity on the germination of the two weeds. Similarly, Evangeline et al. (2012) and Manimegalai (2013) reported allelopathic effects of Tectona grandis on the germination and seedling growth of Vigna mungo and Vigna radiata respectively.

Given the huge economic benefits of T. grandis, which has made it a largely sought after species of wood across the world including Nigeria, the possibility, therefore, exists for overexploitation of this forest resource. As such, many timber farmers may popularize their plantations with Teak. One of the major advantages of relying on the tree for agroforestry interventions over a wide area or climate is because $T$. grandis will survive and grow under a wide range of climatic and edaphic conditions. The question, therefore, is whether teak plantations would impact negatively on the distribution and diversity of other plants as well as the soil characteristics of the area. Although studies related to T. grandis have been carried out across other countries of the world including Nigeria (Akindele, 1989, Aborisade and Aweto 1990, Izekor and Fuwape 2011, Oyebade and Anaba 2018), not much is known about the single-tree influence of teak on plant diversity. The aim of this study, therefore, was to investigate the effects of teak plantation on plant species diversity within and around the tree, as well as the impacts on soil physicochemical characteristics.

\section{Methods}

\section{Study area}

The study was carried out at the Moist Forest Research Station, Benin City located along Utagban road, Off Ekehuan Road (6 $\left.64^{\circ} 4^{\prime} 0^{\prime} \mathrm{N}, 5^{\circ} 34^{\prime \prime} 34^{\prime} \mathrm{E}\right)$. It is a reserve measuring $1 \mathrm{mi}^{2}$ (about $258.999 \mathrm{ha}$ ) jotting towards the Ogba river behind Airport road. The landmass is a reserve that was endowed with various exotic and indigenous forest tree species such as Khaya sp., Lovoa trichilioides, Nauclea diderrichii, Allanblackia floribunda, just to mention a few, as well as a wide array of animals, including reptiles, birds and mammals species 
before the forest was clear-felled; which lead to rigorous replanting/reforestation by successive administrations of which a Tectona grandis plantation was established measuring about $45.72 \mathrm{~m}$ by $91.44 \mathrm{~m}$ from which our study was carried. The forest was planted in the year 2011. Routine clearing of undergrowth in the forest occurs annually, usually during the dry season in other to forestall any outbreak of fire.

\section{Sampling method and procedure}

For the purpose of this study, $36.57 \mathrm{~m}$ by $60.96 \mathrm{~m}$ was marked out of the teak plantation using a measuring tape. The marked out area was divided into three columns with five rows making 15 equal sized subplots measuring $12.19 \mathrm{~m}$ by $12.19 \mathrm{~m}$ each. From the 15 subplots, five subplots were randomly selected from each of the rows. Each subplot contained an average of ten trees per plot. In each of the randomly selected subplot, only one of the trees within each of the subplot was used for the experiment. The five selected plots were pegged using small pegs not more than $0.91 \mathrm{~m}$, labelled with Mon Ami black permanent markers and demarcated into $0-0.5 \mathrm{~m}, 0.5-1.0 \mathrm{~m}$ and $1.0-1.5 \mathrm{~m}$, respectively from the base of the tree using white twines. The trees used in this study were thereafter measured. The subplots were demarcated using ranging poles, pegs, and twines. Soil samples were taken using a soil auger.

\section{Data collection}

Measurements of heights, girths and canopy heights were taken. The height of the tree and the canopy height were measured using Haga altimeter, while the girth was measured using a metre tape. A $1 \mathrm{~m}$ by $1 \mathrm{~m}$ quadrat was thrown on the subplots to identify species diversity and population count. A stem count of the flora available within the study area was used in identifying and counting the species. Soil samples were collected using soil auger within and within and beyond the canopy fringes of the trees in the study area at a depth of $10 \mathrm{~cm}$ from the soil surface and taken to the laboratory in a clean black polythene bag for analysis.

\section{Laboratory analysis and identification of flora species}

The soil physicochemical parameters were analysed at a laboratory following standard procedures (Bray and Kurtz 1945a,b, SSSA 1971, Haluschak 2006, ICARDA 2013, Nasir et al. 2015). The flora species collected were identified with the assistance of the Plant Taxonomists at both the Moist Forest Research Station, Benin City (Forestry Research Institute of Nigeria), and the Department of Plant Biology and Biotechnology, University of Benin, Nigeria. A plant identification text was also used where necessary (Akobundu and Agyakwa, 1998).

\section{Data analysis}

Plant abundance within and outside canopy demarcations was analysed using the IBM Statistical Package for Social Sciences version 20.0 for Windows (SPSS v.20). Correlation, mean, standard deviation and variances were the analytical parameters considered. SPSS was also used to compare soil physicochemical parameters and species abundance within and around the tree canopy. To analyse the flora species collected, diversity indices (Taxa, Dominance, Simpson, Shannon-Winner, Evenness, Brillouin, Menhinick, Margalef, Equitability, BegerParker and Chao-1) were used. These were analysed using the statistical software called $\mathrm{PAST}^{\circ}$ version $2.17 \mathrm{c}$. Mean, range and standard deviation were the descriptive tools considered.

\section{Results}

The morphological characteristics of $T$. grandis have been presented in Table 1. Plant height averaged $11.8 \mathrm{~m}$ whereas canopy length averaged $2.41 \mathrm{~m}$. The highest level of variability amongst the trees sampled occurred with stem girth $(\mathrm{CV}=11.99)$ compared to the other tree parameters measured.

The soil physicochemical parameter was determined around the region covered by the canopy as well as beyond its fringes (Table 2). The $\mathrm{pH}$ was significantly lower under tree canopy ( $\mathrm{pH}$ 4.4) compared to outside the canopy demarcation ( $\mathrm{pH}$ 5.4). However, in spite of the minimal differences in electric conductivity (EC) between the two soil areas, no significant differences were observed $(p<0.05)$. Similarly, no difference between soil composition of calcium $(15.2-17.3 \mathrm{meq} / 100 \mathrm{~g})$, potassium (1.1-1.4 meq/100 g), magnesium (13.4-18.2 meq/ $100 \mathrm{~g})$ and Sulphate $(14.9-18.6 \mathrm{mg} / \mathrm{kg}$ ) were reported in the soil samples collected with the subplot, whether close or far from the tree base. Total organic carbon and total nitrogen within $1.5 \mathrm{~m}$ from the tree base were significantly higher than beyond (Table 2). As reported earlier, 5 subplots (Q1-Q5) within the forest were randomly selected. Each subplot contained at least 20 plants out of the 36 identified in the forest; including Eleusine indica, Cynodon dactylon, Axonopus compressus and Oplismenus burmannii. However, Aneilema beniniense, Sida garckeana, Reisantia indica, Mallotus oppositifolius,

Table 1 Mensuration of the Tectona grandis stands

\begin{tabular}{|c|c|c|c|c|c|c|c|c|}
\hline \multirow{2}{*}{$\begin{array}{l}\text { Plant } \\
\text { parameters } \\
\text { (m) }\end{array}$} & \multicolumn{5}{|c|}{ Subplots } & \multirow[t]{2}{*}{ Mean } & \multirow[t]{2}{*}{ SD } & \multirow[t]{2}{*}{ CV } \\
\hline & Q.1 & Q.2 & Q.3 & Q.4 & Q.5 & & & \\
\hline Height & 12 & 11 & 11.5 & 12.5 & 12 & 11.80 & 0.57 & 4.83 \\
\hline Girth & 0.5 & 0.4 & 0.47 & 0.55 & 0.53 & 0.49 & 0.06 & 11.99 \\
\hline Canopy height & 2.45 & 2.14 & 2.46 & 2.56 & 2.43 & 2.41 & 0.16 & 6.56 \\
\hline
\end{tabular}

Q1-Q5 represent each of the five subplots

$S D$ standard deviation, $C V$ coefficient of variation 
Table 2 Physicochemical parameter of soil within each designated subplot in the forest

\begin{tabular}{|c|c|c|c|c|c|c|c|}
\hline \multirow[t]{2}{*}{ Test variable } & \multirow[t]{2}{*}{ Condition } & \multirow[t]{2}{*}{ Mean } & \multirow[t]{2}{*}{ SD } & \multirow[t]{2}{*}{$t$ value } & \multirow{2}{*}{$\begin{array}{l}p \\
\text { value }\end{array}$} & \multicolumn{2}{|l|}{$95 \% \mathrm{Cl}$} \\
\hline & & & & & & Lower & Upper \\
\hline \multirow[t]{2}{*}{$\mathrm{pH}$} & W1.5m & 4.4 & 0.3 & -4.348 & $0.003^{*}$ & -1.41 & -0.42 \\
\hline & Out1.5 m & 5.4 & 0.4 & & & & \\
\hline \multirow[t]{2}{*}{ Electric conduct ( $\mu \mathrm{s} / \mathrm{cm})$} & W1.5m & 324.8 & 40.4 & 1.185 & 0.270 & -22.13 & 68.93 \\
\hline & Out1.5 m & 301.4 & 17.9 & & & & \\
\hline \multirow[t]{2}{*}{ Total organic carbon (\%) } & W1.5m & 0.8 & 0.3 & 3.164 & $0.013^{*}$ & 0.11 & 0.69 \\
\hline & Out1.5 m & 0.4 & 0.1 & & & & \\
\hline \multirow[t]{2}{*}{ Total nitrogen (\%) } & W1.5m & 0.3 & 0.1 & 2.753 & $0.025^{*}$ & 0.02 & 0.18 \\
\hline & Out1.5 m & 0.2 & 0.0 & & & & \\
\hline \multirow[t]{2}{*}{ Potassium (meq/100 g soil) } & W1.5m & 1.1 & 0.2 & -1.715 & 0.125 & -0.83 & 0.12 \\
\hline & Out1.5 m & 1.4 & 0.4 & & & & \\
\hline \multirow[t]{2}{*}{ Calcium (meq/100 g soil) } & W1.5m & 15.2 & 2.7 & -1.010 & 0.342 & -6.84 & 2.67 \\
\hline & Out1.5 m & 17.3 & 3.7 & & & & \\
\hline \multirow[t]{2}{*}{ Magnessium (meq/100 g soil) } & W1.5m & 18.2 & 6.2 & 1.645 & 0.139 & -1.94 & 11.62 \\
\hline & Out1.5 m & 13.4 & 2.3 & & & & \\
\hline \multirow[t]{2}{*}{ Soluble phosphorus (mg/kg) } & W1.5m & 187.6 & 27.9 & 2.689 & $0.028^{*}$ & 5.11 & 66.61 \\
\hline & Out1.5 m & 151.7 & 10.6 & & & & \\
\hline \multirow[t]{2}{*}{ Sulphate (mg/kg) } & W1.5m & 18.6 & 4.7 & 1.687 & 0.130 & -1.34 & 8.68 \\
\hline & Out1.5 m & 14.9 & 1.2 & & & & \\
\hline
\end{tabular}

W1.5m within $1.5 \mathrm{~m}$ from base, Out $1.5 \mathrm{~m}$ outside canopy demarcation, $S D$ standard deviation, $\mathrm{Cl}$ confidence interval

Euphorbia hirta, Alchornea laxiflora, Tridax procumbens, Chromolaena odorata, Ageratum conyzoides, Panicum laxum, Ludwigia abyssinica, Setaria barbata and Sorghum arandinaceum were absent within $0.5 \mathrm{~m}$ from the tress base (Table 3).

Within the distance of $0.5-1.0 \mathrm{~m}$ from the base of the tree, there were a total of 26 plants identified of different species totaling 179 (Table 4). As with the previous demarcations (0-0.5 m), Reisantia indica, Euphorbia hirta, Tridax procumbens, Chromolaena odorata were also absent (Table 4). Plant distribution under the canopy from within the $1.0-1.5 \mathrm{~m}$ radial demarcation included a total of 762 individual plants species comprising of 28 taxa (Table 5); these included Eleusine indica, Cynodon dactylon, Axonopus compressus, Anthropogon gayanus, Tridax procumbens, Snydrella nodiflora and Smilax anceps respectively. Comparing the results of total plant species counted within the 3 demarcations under the tree canopy, it was generally observed that the totality of individual plant species increased further away from the base of the tree. Within the 1.0-1.5-m space, Commelina diffusa, Aneilema beniniense and Aspilia Africana had the highest coefficient of variability amongst the plants discovered. The totality of plant species counted within the entire subplots showed an average of 398 Cynodon dactylon plant species per plot and 215 Panicum maximum species per plot (Table 5).
As shown in Table 6, Triumfetta cordifolia was the fewest plant species within the subplots and was only found in 1 of 5 subplots. However, Eleusine indica, Cynodon dactylon, Axonopus compressus , Commelina diffusa, Aneilema aequinoctiale, Sida garckeana, Schrankia leptocarpa, Anthonotha macrophylla, Reisantia indica, Brachiaria deflexa, Mallotus oppositifious, Euphorbia hirta, Alchornea laxiflora, Alchonea cordifolia, Combretum hispidum, Newbouldia laevis, Tridax procumbens, Synedrella nodiflora, Chromolaena odorata, Gomphrena celosiodes, Panicum laxum, Ludwigia abyssinica, Icacina trichantha, Oplimenus burmanii, Paspalum conjugatum, Setaria barbata, Phylanthus amarus, Sorghum arandinaceum and Smilax anceps were represented in at least 4 of 5 subplots

The percentage of plants abundance within specified distances from the tree base and under the canopy was compared with the totals obtained within the respective subplots and expressed on a percentage (Table 7). E. indica had a $7.03 \%$ relative abundance at $0.5 \mathrm{~m}$ from the tree base, and $9.83 \%$ further away from the tree, and then $10.16 \%$ at the $1.0-1.5-\mathrm{m}$ radial distance from the tree. This was the same for Combretum hispidum, Newbouldia laevis, Gomphrena celoiodes, Aspilia Africana, Ludwigia abyssinica, Oplismenus burmannii, Paspalum conjugatum, Stetera barbata, and Phylantus amarus. However, the relative abundance of Smilax aceps, Schrankia leptocarpa and Icacina trichantha was highest 
Table 3 Plant distribution at radial distance of $0.5 \mathrm{~m}$ from trunk of tree (under canopy)

\begin{tabular}{|c|c|c|c|c|c|c|c|c|c|c|c|c|}
\hline \multirow{2}{*}{$\begin{array}{l}\mathrm{S} / \\
\mathrm{N}\end{array}$} & \multirow[t]{2}{*}{ Plant identity } & \multirow[t]{2}{*}{ Common name } & \multirow[t]{2}{*}{ Family } & \multicolumn{5}{|c|}{ Within selected tree $(0-0.5 \mathrm{~m})$} & \multirow[t]{2}{*}{ Sum } & \multirow[t]{2}{*}{ Mean } & \multirow[t]{2}{*}{ SD } & \multirow[t]{2}{*}{ CV } \\
\hline & & & & $\overline{Q .1}$ & Q.2 & Q.3 & Q.4 & $\overline{Q .5}$ & & & & \\
\hline 1 & Eleusine indica & Goose grass & Poaceae & 3 & 1 & 0 & 4 & 1 & 9 & 1.8 & 1.6 & 91 \\
\hline 2 & Cynodon dactylon & Bahama grass & Poaceae & 6 & 15 & 12 & 28 & 6 & 67 & 13 & 9 & 68 \\
\hline 3 & Axonopus compressus & Broad leaf carpet grass & Poaceae & 0 & 0 & 0 & 5 & 0 & 5 & 1 & 2.2 & 224 \\
\hline 4 & Anthropogon gayanus & Gamba grass & Poaceae & 2 & 0 & 0 & 1 & 2 & 5 & 1 & 1 & 100 \\
\hline 5 & Commelina diffusa & Spreading day flower & Commelinaceae & 0 & 0 & 0 & 0 & 2 & 2 & 0.4 & 0.9 & 224 \\
\hline 6 & Aneilema beniniense & - & Commelinaceae & 0 & 0 & 0 & 0 & 0 & 0 & 0 & 0 & 0 \\
\hline 7 & Aneilema aequinoctiale & - & Commelinaceae & 0 & 3 & 0 & 0 & 0 & 3 & 0.6 & 1.3 & 224 \\
\hline 8 & Triumfetta cordifolia & - & Malvaceae & 0 & 0 & 0 & 0 & 3 & 3 & 0.6 & 1.3 & 224 \\
\hline 9 & Sida garckeana & - & Malvaceae & 0 & 0 & 0 & 0 & 0 & 0 & 0 & 0 & 0 \\
\hline 10 & Schrankia leptocarpa & Sentifure plant & Fabaceae & 0 & 0 & 0 & 6 & 0 & 6 & 1.2 & 2.7 & 224 \\
\hline 11 & Daniella oliveri & Ilorin basam & Fabaceae & 0 & 0 & 0 & 1 & 0 & 1 & 0.2 & 0.4 & 224 \\
\hline 12 & Anthonotha macrophylla & - & Fabaceae & 0 & 1 & 1 & 1 & 7 & 10 & 2 & 2.8 & 14 \\
\hline 13 & Reissantia indica & Wild halle & Hippocrateaceae & 0 & 0 & 0 & 0 & 0 & 0 & 0 & 0 & 0 \\
\hline 14 & Brachiaria deflexa & - & Poaceae & 0 & 0 & 4 & 0 & 0 & 4 & 0.8 & 1.8 & 224 \\
\hline 15 & Mallotus oppositifolius & - & Euphorbiaceae & 0 & 0 & 0 & 0 & 0 & 0 & 0 & 0 & 0 \\
\hline 16 & Euphorbia hirta & Autralian asthma plant & Euphorbiaceae & 0 & 0 & 0 & 0 & 0 & 0 & 0 & 0 & 0 \\
\hline 17 & Alchornea laxiflora & - & Euphorbiaceae & 0 & 0 & 0 & 0 & 0 & 0 & 0 & 0 & 0 \\
\hline 18 & Alchornea cordifolia & Christmas bush & Euphorbiaceae & 0 & 0 & 0 & 0 & 1 & 1 & 0.2 & 0.4 & 224 \\
\hline 19 & Momordica charantia & African cucumber & Cucurbitaceae & 0 & 0 & 0 & 0 & 0 & 0 & 0 & 0 & 0 \\
\hline 20 & Combretum hispidum & - & Combretaceae & 1 & 1 & 2 & 1 & 0 & 5 & 1 & 0.7 & 71 \\
\hline 21 & Newbouldia laevis & - & Bignoniaceaea & 0 & 0 & 0 & 0 & 1 & 1 & 0.2 & 0.4 & 224 \\
\hline 22 & Tridax procumbens & Tridax & Asteraceae & 0 & 0 & 0 & 0 & 0 & 0 & 0 & 0 & 0 \\
\hline 23 & Synedrella nodiflora & Nodeweed & Asteraceae & 0 & 5 & 3 & 5 & 0 & 13 & 2.6 & 2.5 & 97 \\
\hline 24 & Chromolaena odorata & Siam weed & Asteraceae & 0 & 0 & 0 & 0 & 0 & 0 & 0 & 0 & 0 \\
\hline 25 & Ageratum conyzoides & Billy goat wed & Asteraceae & 0 & 0 & 0 & 0 & 0 & 0 & 0 & 0 & 0 \\
\hline 26 & Gomphrena celosioides & - & Amaranthaceae & 1 & 0 & 0 & 0 & 1 & 2 & 0.4 & 0.5 & 137 \\
\hline 27 & Panicum laxum & Panic grass & Poaceae & 0 & 0 & 0 & 0 & 0 & 0 & 0 & 0 & 0 \\
\hline 28 & Aspilia Africana & - & Asteraceae & 0 & 0 & 0 & 0 & 1 & 1 & 0.2 & 0.4 & 224 \\
\hline 29 & Ludwigia abyssinica & Water primus & Onograceae & 0 & 0 & 0 & 0 & 0 & 0 & 0 & 0 & 0 \\
\hline 30 & Icacina trichantha & - & Liacinaceae & 0 & 1 & 3 & 1 & 0 & 5 & 1 & 1.2 & 123 \\
\hline 31 & Oplismenus burmannii & - & Poaceae & 4 & 0 & 10 & 0 & 5 & 19 & 3.8 & 4.1 & 109 \\
\hline 32 & Paspalum conjugatum & Sour grass & Poaceae & 1 & 1 & 1 & 1 & 0 & 4 & 0.8 & 0.4 & 56 \\
\hline 33 & Setaria barbata & Brisky toxtail & Poaceae & 0 & 0 & 0 & 0 & 0 & 0 & 0 & 0 & 0 \\
\hline 34 & Phyllanthus amarus & - & Poaceae & 0 & 0 & 1 & 0 & 0 & 1 & 0.2 & 0.4 & 224 \\
\hline 35 & Sorghum arundinaceum & - & Poaceae & 0 & 0 & 0 & 0 & 0 & 0 & 0 & 0 & 0 \\
\hline 36 & Smilax anceps & - & Poaceae & 2 & 1 & 6 & 1 & 2 & 12 & 2.4 & 2.1 & 86 \\
\hline
\end{tabular}

$S D$ standard deviation, $C V$ coefficient of variation

when the plants were closer to the tree base than further away; thereby suggesting possible rhizospheric influence of T. grandis. Aneilema beniniense, Sida garckeana, Reisantia indica, Mallotus oppositifolius, Euphorbia hirta, Alchornea laxiflora, Momoedceae chrantia, Tridax procumbens, Chromolaena odorata, Ageratum conyzoides, Panicum laxum, Ludwigia abyssinica, Stetera barbata and Sorghum arandinaceum were all absent within 0.5 $\mathrm{m}$ from the tree base; perhaps suggesting inhibitory rhizospheric influence.

Statistical differences between plant abundance within and outside canopy demarcations have been presented (Table 8). For Eleusine indica, plant abundance under the canopy and outside canopy demarcations were statistically 
Table 4 Plant distribution at radial distance of $0.5-1.0 \mathrm{~m}$ from trunk of tree (under canopy)

\begin{tabular}{|c|c|c|c|c|c|c|c|c|c|c|}
\hline \multirow{2}{*}{$\begin{array}{l}\mathrm{S} / \\
\mathrm{N}\end{array}$} & \multirow[t]{2}{*}{ Plant identity } & \multicolumn{5}{|c|}{ Within selected tree $(0.5-1.0 \mathrm{~m})$} & \multirow[b]{2}{*}{ Sum } & \multirow[b]{2}{*}{ Mean } & \multirow[b]{2}{*}{ SD } & \multirow[b]{2}{*}{$C V$} \\
\hline & & Q.1 & Q.2 & Q.3 & Q.4 & Q.5 & & & & \\
\hline 1 & Eleusine indica & 2 & 6 & 0 & 2 & 2 & 12 & 2.4 & 2.2 & 91.3 \\
\hline 2 & Cynodon dactylon & 17 & 50 & 27 & 11 & 21 & 126 & 25.2 & 15.0 & 59.7 \\
\hline 3 & Axonopus compressus & 2 & 2 & 2 & 3 & 2 & 11 & 2.2 & 0.4 & 20.3 \\
\hline 4 & Anthropogon gayanus & 3 & 4 & 3 & 4 & 3 & 17 & 3.4 & 0.5 & 16.1 \\
\hline 5 & Commelina diffusa & 0 & 0 & 0 & 0 & 0 & 0 & 0 & 0.0 & 0.0 \\
\hline 6 & Aneilema beniniense & 2 & 0 & 0 & 6 & 0 & 8 & 1.6 & 2.6 & 163.0 \\
\hline 7 & Aneilema aequinoctiale & 0 & 1 & 0 & 9 & 0 & 10 & 2 & 3.9 & 196.9 \\
\hline 8 & Triumfetta cordifolia & 0 & 0 & 0 & 0 & 0 & 0 & 0 & 0.0 & 0.0 \\
\hline 9 & Sida garckeana & 0 & 0 & 0 & 1 & 0 & 1 & 0.2 & 0.4 & 223.6 \\
\hline 10 & Schrankia leptocarpa & 0 & 0 & 0 & 0 & 0 & 0 & 0 & 0.0 & 0.0 \\
\hline 11 & Daniella oliveri & 0 & 0 & 0 & 5 & 0 & 5 & 1 & 2.2 & 223.6 \\
\hline 12 & Anthonotha macrophylla & 0 & 1 & 1 & 3 & 0 & 5 & 1 & 1.2 & 122.5 \\
\hline 13 & Reissantia indica & 0 & 0 & 0 & 0 & 0 & 0 & 0 & 0.0 & 0.0 \\
\hline 14 & Brachiaria deflexa & 10 & 10 & 10 & 10 & 9 & 49 & 9.8 & 0.4 & 4.6 \\
\hline 15 & Mallotus oppositifolius & 0 & 0 & 0 & 0 & 9 & 9 & 1.8 & 4.0 & 223.6 \\
\hline 16 & Euphorbia hirta & 0 & 0 & 0 & 0 & 0 & 0 & 0 & 0.0 & 0.0 \\
\hline 17 & Alchornea laxiflora & 3 & 3 & 3 & 3 & 0 & 12 & 2.4 & 1.3 & 55.9 \\
\hline 18 & Alchornea cordifolia & 1 & 0 & 0 & 0 & 1 & 2 & 0.4 & 0.5 & 136.9 \\
\hline 19 & Momordica charantia & 0 & 0 & 0 & 0 & 0 & 0 & 0 & 0.0 & 0.0 \\
\hline 20 & Combretum hispidum & 0 & 0 & 3 & 0 & 0 & 3 & 0.6 & 1.3 & 223.6 \\
\hline 21 & Newbouldia laevis & 0 & 3 & 0 & 3 & 0 & 6 & 1.2 & 1.6 & 136.9 \\
\hline 22 & Tridax procumbens & 0 & 0 & 0 & 0 & 0 & 0 & 0 & 0.0 & 0.0 \\
\hline 23 & Synedrella nodiflora & 10 & 2 & 7 & 2 & 4 & 25 & 5 & 3.5 & 69.3 \\
\hline 24 & Chromolaena odorata & 0 & 0 & 0 & 0 & 0 & 0 & 0 & 0.0 & 0.0 \\
\hline 25 & Ageratum conyzoides & 0 & 0 & 0 & 0 & 4 & 4 & 0.8 & 1.8 & 223.6 \\
\hline 26 & Gomphrena celosioides & 0 & 3 & 1 & 3 & 0 & 7 & 1.4 & 1.5 & 108.3 \\
\hline 27 & Panicum laxum & 0 & 0 & 0 & 0 & 0 & 0 & 0 & 0.0 & 0.0 \\
\hline 28 & Aspilia Africana & 0 & 1 & 1 & 2 & 0 & 4 & 0.8 & 0.8 & 104.6 \\
\hline 29 & Ludwigia abyssinica & 1 & 1 & 0 & 3 & 4 & 9 & 1.8 & 1.6 & 91.3 \\
\hline 30 & Icacina trichantha & 0 & 0 & 2 & 0 & 0 & 2 & 0.4 & 0.9 & 223.6 \\
\hline 31 & Oplismenus burmannii & 12 & 17 & 30 & 11 & 16 & 86 & 17.2 & 7.6 & 44.2 \\
\hline 32 & Paspalum conjugatum & 3 & 5 & 5 & 2 & 0 & 15 & 3 & 2.1 & 70.7 \\
\hline 33 & Setaria barbata & 1 & 0 & 0 & 2 & 3 & 6 & 1.2 & 1.3 & 108.7 \\
\hline 34 & Phyllanthus amarus & 0 & 0 & 0 & 3 & 0 & 3 & 0.6 & 1.3 & 223.6 \\
\hline 35 & Sorghum arundinaceum & 0 & 0 & 0 & 0 & 0 & 0 & 0 & 0.0 & 0.0 \\
\hline 36 & Smilax anceps & 0 & 3 & 2 & 4 & 0 & 9 & 1.8 & 1.8 & 99.4 \\
\hline
\end{tabular}

similar; implying that the tree may not have significantly affected plant distribution. Species abundance of Cynodon dactylon, Axonopus compressus, Anthropogon gayanus, Commelina diffusa, Aneilema beniniense, Aneilema aequinoctiale, Sida garckeana, Anthonotha macrophylla, Reisantia indica and Euphorbia hirta were generally suppressed.
Diversity indices of plant species within and outside canopy demarcations were compared (Table 9). Generally, there were fewer species within $1.5 \mathrm{~m}$ from the tree than beyond this radial demarcation, thus indicating inhibitory effects of tree presence. The implication of this suppressed species abundance within close proximity to the tree is the possibility for a number of dominant species to spring up 
Table 5 Plant distribution at radial distance of 1.0-1.5 m from trunk of tree (under canopy)

\begin{tabular}{|c|c|c|c|c|c|c|c|c|c|c|}
\hline \multirow{2}{*}{$\begin{array}{l}\mathrm{S} / \\
\mathrm{N}\end{array}$} & \multirow[t]{2}{*}{ Plant identity } & \multicolumn{5}{|c|}{ Within selected tree $(1.0-1.5 \mathrm{~m})$} & \multirow[b]{2}{*}{ Sum } & \multirow[b]{2}{*}{ Mean } & \multirow[b]{2}{*}{ SD } & \multirow[b]{2}{*}{ CV } \\
\hline & & Q.1 & Q.2 & Q.3 & Q.4 & Q.5 & & & & \\
\hline 1 & Eleusine indica & 2 & 4 & 0 & 5 & 2 & 13 & 2.6 & 1.9 & 75.0 \\
\hline 2 & cynodon dactylon & 30 & 50 & 36 & 34 & 14 & 164 & 32.8 & 12.9 & 39.4 \\
\hline 3 & Axonopus compressus & 3 & 0 & 0 & 0 & 3 & 6 & 1.2 & 1.6 & 136.9 \\
\hline 4 & Anthropogon gayanus & 6 & 7 & 8 & 7 & 6 & 34 & 6.8 & 0.8 & 12.3 \\
\hline 5 & Commelina diffusa & 0 & 0 & 0 & 4 & 0 & 4 & 0.8 & 1.8 & 223.6 \\
\hline 6 & Aneilema beniniense & 0 & 0 & 0 & 8 & 0 & 8 & 1.6 & 3.6 & 223.6 \\
\hline 7 & Aneilema aequinoctiale & 1 & 0 & 0 & 4 & 1 & 6 & 1.2 & 1.6 & 136.9 \\
\hline 8 & Triumfetta cordifolia & 0 & 0 & 0 & 0 & 0 & 0 & 0 & 0.0 & 0.0 \\
\hline 9 & Sida garckeana & 0 & 0 & 0 & 0 & 0 & 0 & 0 & 0.0 & 0.0 \\
\hline 10 & Schrankia leptocarpa & 0 & 0 & 0 & 0 & 0 & 0 & 0 & 0.0 & 0.0 \\
\hline 11 & Daniella oliveri & 0 & 0 & 0 & 0 & 0 & 0 & 0 & 0.0 & 0.0 \\
\hline 12 & Anthonotha macrophylla & 3 & 1 & 1 & 1 & 3 & 9 & 1.8 & 1.1 & 60.9 \\
\hline 13 & Reissantia indica & 0 & 0 & 0 & 0 & 0 & 0 & 0 & 0.0 & 0.0 \\
\hline 14 & Brachiaria deflexa & 10 & 10 & 115 & 10 & 10 & 155 & 31 & 47.0 & 151.5 \\
\hline 15 & Mallotus oppositifolius & 0 & 0 & 0 & 0 & 0 & 0 & 0 & 0.0 & 0.0 \\
\hline 16 & Euphorbia hirta & 0 & 3 & 0 & 3 & 0 & 6 & 1.2 & 1.6 & 136.9 \\
\hline 17 & Alchornea laxiflora & 10 & 10 & 10 & 10 & 10 & 50 & 10 & 0.0 & 0.0 \\
\hline 18 & Alchornea cordifolia & 8 & 5 & 5 & 5 & 4 & 27 & 5.4 & 1.5 & 28.1 \\
\hline 19 & Momordica charantia & 0 & 0 & 0 & 0 & 0 & 0 & 0 & 0.0 & 0.0 \\
\hline 20 & Combretum hispidum & 5 & 2 & 2 & 2 & 5 & 16 & 3.2 & 1.6 & 51.3 \\
\hline 21 & Newbouldia laevis & 3 & 0 & 1 & 0 & 5 & 9 & 1.8 & 2.2 & 120.4 \\
\hline 22 & Tridax procumbens & 0 & 1 & 0 & 1 & 0 & 2 & 0.4 & 0.5 & 136.9 \\
\hline 23 & Synedrella nodiflora & 3 & 5 & 5 & 5 & 2 & 20 & 4 & 1.4 & 35.4 \\
\hline 24 & Chromolaena odorata & 0 & 1 & 0 & 1 & 5 & 7 & 1.4 & 2.1 & 148.1 \\
\hline 25 & Ageratum conyzoides & 3 & 0 & 0 & 1 & 6 & 10 & 2 & 2.5 & 127.5 \\
\hline 26 & Gomphrena celosioides & 4 & 5 & 2 & 5 & 0 & 16 & 3.2 & 2.2 & 67.7 \\
\hline 27 & Panicum laxum & 5 & 6 & 6 & 12 & 0 & 29 & 5.8 & 4.3 & 73.6 \\
\hline 28 & Aspilia Africana & 0 & 0 & 0 & 0 & 2 & 2 & 0.4 & 0.9 & 223.6 \\
\hline 29 & Ludwigia abyssinica & 2 & 4 & 0 & 5 & 2 & 13 & 2.6 & 1.9 & 75.0 \\
\hline 30 & Icacina trichantha & 0 & 0 & 0 & 0 & 0 & 0 & 0 & 0.0 & 0.0 \\
\hline 31 & Oplismenus burmannii & 15 & 10 & 60 & 10 & 3 & 98 & 19.6 & 23.0 & 117.3 \\
\hline 32 & Paspalum conjugatum & 1 & 8 & 8 & 8 & 9 & 34 & 6.8 & 3.3 & 48.1 \\
\hline 33 & Setaria barbata & 0 & 1 & 5 & 1 & 3 & 10 & 2 & 2.0 & 100.0 \\
\hline 34 & Phyllanthus amarus & 0 & 0 & 3 & 2 & 0 & 5 & 1 & 1.4 & 141.4 \\
\hline 35 & Sorghum arundinaceum & 0 & 0 & 0 & 3 & 0 & 3 & 0.6 & 1.3 & 223.6 \\
\hline 36 & Smilax anceps & 3 & 0 & 0 & 0 & 3 & 6 & 1.2 & 1.6 & 136.9 \\
\hline
\end{tabular}

around the tree canopy. With a Brillouin index of 2.941 beyond the canopy demarcation and 2.601 within the canopy, it was suggested that the group diversity of plant species outside the $1.5-\mathrm{m}$ demarcation was slightly higher than within. However, going by the Berger-parkerindex value of 0.258 under tree canopy (UC) compared to 0.190 beyond the demarcation $(\mathrm{BC})$, the dominant species within
$1.5 \mathrm{~m}$ from the tree were more abundant than those in beyond (Table 9). There was a highly significant negative correlation between species abundance and total organic carbon of the soil outside the tree canopy $(R=-0.880, p<$ $0.05)$ (Table 10). Similarly, species index also negatively correlated with soil sulphates $(R=-0.906)$ at spaces beyond $1.5 \mathrm{~m}$ from the tree. Species abundance outside the $1.5-\mathrm{m}$ 
Table 6 Plant distribution with each quadrant, inclusive of vegetative counts about the test tree

\begin{tabular}{|c|c|c|c|c|c|c|c|c|c|c|}
\hline \multirow{2}{*}{$\begin{array}{l}\mathrm{S} / \\
\mathrm{N}\end{array}$} & \multirow[t]{2}{*}{ Plant identity } & \multicolumn{5}{|c|}{ Within subplot abundance } & \multirow[b]{2}{*}{ Sum } & \multirow[b]{2}{*}{ Mean } & \multirow[b]{2}{*}{ SD } & \multirow[b]{2}{*}{$C V$} \\
\hline & & Q.1 & Q.2 & Q.3 & Q.4 & Q.5 & & & & \\
\hline 1 & Eleusine indica & 10 & 50 & 0 & 58 & 10 & 128 & 25.6 & 26.4 & 103.1 \\
\hline 2 & cynodon dactylon & 300 & 600 & 150 & 518 & 421 & 1989 & 398 & 178.1 & 44.8 \\
\hline 3 & Axonopus compressus & 20 & 20 & 20 & 20 & 43 & 123 & 24.6 & 10.3 & 41.8 \\
\hline 4 & Anthropogon gayanus & 70 & 50 & 150 & 50 & 58 & 378 & 75.6 & 42.4 & 56.1 \\
\hline 5 & Commelina diffusa & 13 & 10 & 40 & 11 & 44 & 118 & 23.6 & 16.9 & 71.6 \\
\hline 6 & Aneilema beniniense & 38 & 54 & 123 & 43 & 37 & 295 & 59 & 36.4 & 61.7 \\
\hline 7 & Aneilema aequinoctiale & 45 & 56 & 0 & 56 & 54 & 211 & 42.2 & 24.0 & 56.9 \\
\hline 8 & Triumfetta cordifolia & 0 & 0 & 0 & 0 & 2 & 2 & 0.4 & 0.9 & 223.6 \\
\hline 9 & Sida garckeana & 10 & 4 & 4 & 4 & 14 & 36 & 7.2 & 4.6 & 63.9 \\
\hline 10 & Schrankia leptocarpa & 0 & 10 & 7 & 10 & 0 & 27 & 5.4 & 5.1 & 94.1 \\
\hline 11 & Daniella oliveri & 0 & 3 & 5 & 3 & 0 & 11 & 2.2 & 2.2 & 98.5 \\
\hline 12 & Anthonotha macrophylla & 12 & 50 & 50 & 50 & 34 & 196 & 39.2 & 16.7 & 42.6 \\
\hline 13 & Reissantia indica & 12 & 13 & 12 & 13 & 0 & 50 & 10 & 5.6 & 56.1 \\
\hline 14 & Brachiaria deflexa & 150 & 150 & 150 & 150 & 150 & 750 & 150 & 0.0 & 0.0 \\
\hline 15 & Mallotus oppositifolius & 100 & 15 & 25 & 15 & 100 & 255 & 51 & 44.9 & 88.1 \\
\hline 16 & Euphorbia hirta & 30 & 15 & 50 & 15 & 30 & 140 & 28 & 14.4 & 51.4 \\
\hline 17 & Alchornea laxiflora & 200 & 200 & 200 & 124 & 200 & 924 & 185 & 34.0 & 18.4 \\
\hline 18 & Alchornea cordifolia & 70 & 60 & 60 & 60 & 70 & 320 & 64 & 5.5 & 8.6 \\
\hline 19 & Momordica charantia & 6 & 0 & 1 & 0 & 6 & 13 & 2.6 & 3.1 & 120.4 \\
\hline 20 & Combretum hispidum & 14 & 15 & 40 & 32 & 14 & 115 & 23 & 12.2 & 53.1 \\
\hline 21 & Newbouldia laevis & 13 & 23 & 13 & 21 & 13 & 83 & 16.6 & 5.0 & 30.0 \\
\hline 22 & Tridax procumbens & 0 & 5 & 14 & 5 & 11 & 35 & 7 & 5.5 & 78.9 \\
\hline 23 & Synedrella nodiflora & 0 & 50 & 70 & 47 & 13 & 180 & 36 & 28.7 & 79.8 \\
\hline 24 & Chromolaena odorata & 25 & 15 & 0 & 22 & 25 & 87 & 17.4 & 10.5 & 60.6 \\
\hline 25 & Ageratum conyzoides & 0 & 0 & 100 & 4 & 21 & 125 & 25 & 42.8 & 171.3 \\
\hline 26 & Gomphrena celosioides & 43 & 24 & 22 & 24 & 43 & 156 & 31.2 & 10.8 & 34.6 \\
\hline 27 & Panicum laxum & 100 & 250 & 550 & 74 & 100 & 1074 & 215 & 199.9 & 93.0 \\
\hline 28 & Aspilia Africana & 0 & 0 & 40 & 8 & 15 & 63 & 12.6 & 16.5 & 131.3 \\
\hline 29 & Ludwigia abyssinica & 8 & 50 & 14 & 64 & 8 & 144 & 28.8 & 26.3 & 91.4 \\
\hline 30 & Icacina trichantha & 21 & 12 & 13 & 12 & 32 & 90 & 18 & 8.7 & 48.3 \\
\hline 31 & Oplismenus burmannii & 100 & 150 & 300 & 84 & 93 & 727 & 145 & 90.1 & 62.0 \\
\hline 32 & Paspalum conjugatum & 120 & 150 & 150 & 25 & 110 & 555 & 111 & 51.3 & 46.2 \\
\hline 33 & Setaria barbata & 15 & 23 & 23 & 55 & 12 & 128 & 25.6 & 17.1 & 67.0 \\
\hline 34 & Phyllanthus amarus & 28 & 22 & 50 & 26 & 32 & 158 & 31.6 & 10.9 & 34.5 \\
\hline 35 & Sorghum arundinaceum & 70 & 56 & 46 & 43 & 54 & 269 & 53.8 & 10.5 & 19.6 \\
\hline 36 & Smilax anceps & 0 & 16 & 40 & 16 & 32 & 104 & 20.8 & 15.6 & 75.0 \\
\hline
\end{tabular}

radial demarcation may have been positively influenced by the soil's organic carbon from soils in close proximity with the tree $(R=0.916, p<0.05)$. The implication of the correlation is that species abundance outside the tree canopy could be enhanced by positively influencing total organic carbon within the canopy or reducing organic carbon outside canopy demarcation.

\section{Discussion}

The results of this study showed that some selected physicochemical characteristics of the soil were influenced by the proximity of the tree to the point of soil collection for analysis.

The $\mathrm{pH}$ of the topsoil $(0-15 \mathrm{~cm})$ obtained randomly within $1.5 \mathrm{~m}$ from the base of the tree was higher than 
Table 7 Relative abundance of plant species within the radial distances within the tree canopy

\begin{tabular}{|c|c|c|c|c|c|}
\hline \multirow{2}{*}{$\begin{array}{l}\mathrm{S} / \\
\mathrm{N}\end{array}$} & \multirow[t]{2}{*}{ Plant identity } & \multirow{2}{*}{$\begin{array}{l}\text { mTotal } \\
\text { (SP) }\end{array}$} & \multicolumn{3}{|c|}{${ }^{* *}$ Relative abundance (\%) } \\
\hline & & & ${ }^{*} 0-0.5 \mathrm{~m}$ & $0.5-1.0 \mathrm{~m}$ & $1.0-1.5 \mathrm{~m}$ \\
\hline 1 & Eleusine indica & 25.6 & 7.03 & 9.38 & 10.16 \\
\hline 2 & cynodon dactylon & 397.8 & 3.37 & 6.33 & 8.25 \\
\hline 3 & Axonopus compressus & 24.6 & 4.07 & 8.94 & 4.88 \\
\hline 4 & Anthropogon gayanus & 75.6 & 1.32 & 4.5 & 8.99 \\
\hline 5 & Commelina diffusa & 23.6 & 1.69 & 0 & 3.39 \\
\hline 6 & Aneilema beniniense & 59 & 0 & 2.71 & 2.71 \\
\hline 7 & Aneilema aequinoctiale & 42.2 & 1.42 & 4.74 & 2.84 \\
\hline 8 & Triumfetta cordifolia & 0.4 & 150 & 0 & 0 \\
\hline 9 & Sida garckeana & 7.2 & 0 & 2.78 & 0 \\
\hline 10 & Schrankia leptocarpa & 5.4 & 22.22 & 0 & 0 \\
\hline 11 & Daniella oliveri & 2.2 & 9.09 & 45.45 & 0 \\
\hline 12 & Anthonotha macrophylla & 39.2 & 5.1 & 2.55 & 4.59 \\
\hline 13 & Reissantia indica & 10 & 0 & 0 & 0 \\
\hline 14 & Brachiaria deflexa & 150 & 0.53 & 6.53 & 20.67 \\
\hline 15 & Mallotus oppositifolius & 51 & 0 & 3.53 & 0 \\
\hline 16 & Euphorbia hirta & 28 & 0 & 0 & 4.29 \\
\hline 17 & Alchornea laxiflora & 184.8 & 0 & 1.3 & 5.41 \\
\hline 18 & Alchornea cordifolia & 64 & 0.31 & 0.63 & 8.44 \\
\hline 19 & Momordica charantia & 2.6 & 0 & 0 & 0 \\
\hline 20 & Combretum hispidum & 23 & 4.35 & 2.61 & 13.91 \\
\hline 21 & Newbouldia laevis & 16.6 & 1.2 & 7.23 & 10.84 \\
\hline 22 & Tridax procumbens & 7 & 0 & 0 & 5.71 \\
\hline 23 & Synedrella nodiflora & 36 & 7.22 & 13.89 & 11.11 \\
\hline 24 & Chromolaena odorata & 17.4 & 0 & 0 & 8.05 \\
\hline 25 & Ageratum conyzoides & 25 & 0 & 3.2 & 8 \\
\hline 26 & Gomphrena celosioides & 31.2 & 1.28 & 4.49 & 10.26 \\
\hline 27 & Panicum laxum & 214.8 & 0 & 0 & 2.7 \\
\hline 28 & Aspilia Africana & 12.6 & 1.59 & 6.35 & 3.17 \\
\hline 29 & Ludwigia abyssinica & 28.8 & 0 & 6.25 & 9.03 \\
\hline 30 & Icacina trichantha & 18 & 5.56 & 2.22 & 0 \\
\hline 31 & Oplismenus burmannii & 145.4 & 2.61 & 11.83 & 13.48 \\
\hline 32 & Paspalum conjugatum & 111 & 0.72 & 2.7 & 6.13 \\
\hline 33 & Setaria barbata & 25.6 & 0 & 4.69 & 7.81 \\
\hline 34 & Phyllanthus amarus & 31.6 & 0.63 & 1.9 & 3.16 \\
\hline 35 & Sorghum arundinaceum & 53.8 & 0 & 0 & 1.12 \\
\hline 36 & Smilax anceps & 20.8 & 11.54 & 8.65 & 5.77 \\
\hline
\end{tabular}

mTotal (SP) mean total of plant species within the subplot *Distance from tree base

**Percentage of plant abundance at distance compared to totals within the subplot

somewhere within and beyond the canopy fringes. This supports the earlier findings of Rhoades (1997), who described that soil $\mathrm{pH}$ under the single-tree influence was lower under canopy than the outside. Another possible explanation for reduced $\mathrm{pH}$ may be in the exudation of organic acids which plants used most times as phytochelators to enhance absorption of nutrients or impede the accumulation of pollutants, as the case may be (Salt et al. 1999). In a similar study by Imoro et al. (2012) in the Afrensu Brohuma Forest Reserve in Ashanti region, Ghana, the authors reported that soil $\mathrm{pH}$ was directly influenced by $T$. grandis $(\mathrm{pH}=7.04)$, when compared with the control plot $(\mathrm{pH}=7.53)$. Watanabe et al. (2009) documented lower $\mathrm{pH}$ values $(\mathrm{pH}=7.14)$.

Kanazawa et al. (1994), Pellet et al. (1995) reported that plants in most iron-contaminated soils usually have need of organic acids that enhance bio-availability of soil-bound iron. The survival of most of these plants in acidic soils also depends on their ability to exude citric and malic acids, amongst other organic acids to chelate the highly phytotoxic rhizospheric $\mathrm{Al}^{3+}$ to form a less toxic complex, a phenomenon which is also common in many oxisols and ultisols; particularly the most predominant soil type in Benin City, Nigeria. Apart from the fact that $\mathrm{Al}^{3+}$ enhances soil acidity (Merino-Gergichevich et al. 2010), the release of organic acids within root zones of the tree to chelate the metal further reduces the soil $\mathrm{pH}$ around this rejoin; perhaps the justification for the reduced $\mathrm{pH}$ reported compared to outside the canopy demarcation.

Although no single mechanism is responsible for changes in soil chemistry, we observed single-tree influence of $T$. grandis was also observed in the soil composition of total organic carbon, total nitrogen and soluble phosphorus. The concentrations of these soil characteristics under the canopy were higher than beyond; also confirming earlier reports (Rhoades 1997, Zinke 1962). The possibility exists therefore that the forest environment probably affect soil nutrients dynamics as earlier suggested by Lal (2005). Imoro et al. (2012) reported that soil nitrogen under the $T$. grandis plantation minimally surpassed that outside the tree plantation.

Enhanced accumulation of organic carbon and phosphorus is most likely attributed to the enormous organic materials, which are consequences of the decay of fallen litter that gathers around the tree. In a number of isolated cases, as observed in the study, some of the foresters, when carrying out routine slashing of the weeds around the planted forest, usually gather most of the weeds and place them around the trunk as mulch. Increasing the quantity of plant material incorporated into the soil usually would further advance soil nutrient standing. Increased organic matter has been reported by Dinakaran and Krishnayya (2010) in teak forested areas.

Single-tree influence in plant association is one of several factors that affect the overall dynamics in agroforestry 
Table 8 Statistical differences between plant abundance within and outside tree canopy

\begin{tabular}{|c|c|c|c|c|c|c|c|c|c|}
\hline \multirow{2}{*}{$\begin{array}{l}\mathrm{S} / \\
\mathrm{N}\end{array}$} & \multirow[t]{2}{*}{ Plant identity } & \multicolumn{3}{|c|}{ Under canopy (< 1.5 m) } & \multicolumn{3}{|c|}{ Outside canopy demarcation (> $1.5 \mathrm{~m}$ ) } & \multirow{2}{*}{$\begin{array}{l}t \\
\text { value }\end{array}$} & \multirow[t]{2}{*}{$p$ value } \\
\hline & & Mean & SD & CV & Mean & SD & CV & & \\
\hline 1 & Eleusine indica & 6 & 5 & 73 & 19 & 484 & 115 & -1.27 & 0.239 \\
\hline 2 & cynodon dactylon & 71 & 28 & 39 & 326 & 27900 & 51 & -3.37 & 0.01 \\
\hline 3 & Axonopus compressus & 4 & 3 & 57 & 20 & 105 & 51 & -3.35 & 0.01 \\
\hline 4 & Anthropogon gayanus & 11 & 0 & 4 & 64 & 1810 & 66 & -2.8 & 0.023 \\
\hline 5 & Commelina diffusa & 1 & 2 & 149 & 22 & 293 & 76 & -2.75 & 0.025 \\
\hline 6 & Aneilema beniniense & 3 & 6 & 191 & 31 & 389 & 63 & -3.03 & 0.016 \\
\hline 7 & Aneilema aequinoctiale & 4 & 5 & 141 & 38 & 481 & 57 & -3.43 & 0.009 \\
\hline 8 & Triumfetta cordifolia & 1 & 1 & 224 & 0 & 0 & 0 & 1 & 0.347 \\
\hline 9 & Sida garckeana & 0 & 0 & 224 & 7 & 23 & 69 & -3.16 & 0.013 \\
\hline 10 & Schrankia leptocarpa & 1 & 3 & 224 & 4 & 19 & 104 & -1.31 & 0.228 \\
\hline 11 & Daniella oliveri & 1 & 3 & 224 & 2 & 5 & 144 & -0.25 & 0.807 \\
\hline 12 & Anthonotha macrophylla & 5 & 3 & 63 & 34 & 296 & 50 & -3.79 & 0.005 \\
\hline 13 & Reissantia indica & 0 & 0 & 0 & 10 & 32 & 56 & -3.98 & 0.004 \\
\hline 14 & Brachiaria deflexa & 42 & 49 & 117 & 108 & 2387 & 45 & -2.16 & 0.063 \\
\hline 15 & Mallotus oppositifolius & 2 & 4 & 224 & 49 & 1813 & 87 & -2.48 & 0.038 \\
\hline 16 & Euphorbia hirta & 1 & 2 & 137 & 27 & 249 & 59 & -3.61 & 0.007 \\
\hline 17 & Alchornea laxiflora & 12 & 1 & 11 & 172 & 1180 & 20 & -10.4 & $<0.001$ \\
\hline 18 & Alchornea cordifolia & 6 & 2 & 29 & 58 & 18 & 7 & -25.4 & $<0.001$ \\
\hline 19 & Momordica charantia & 0 & 0 & 0 & 3 & 10 & 120 & -1.86 & 0.101 \\
\hline 20 & Combretum hispidum & 5 & 2 & 37 & 18 & 141 & 65 & -2.5 & 0.037 \\
\hline 21 & Newbouldia laevis & 3 & 2 & 56 & 13 & 30 & 41 & -3.97 & 0.004 \\
\hline 22 & Tridax procumbens & 0 & 1 & 137 & 7 & 33 & 87 & -2.41 & 0.043 \\
\hline 23 & Synedrella nodiflora & 12 & 3 & 29 & 27 & 525 & 85 & -1.49 & 0.175 \\
\hline 24 & Chromolaena odorata & 1 & 2 & 148 & 16 & 96 & 61 & -3.27 & 0.011 \\
\hline 25 & Ageratum conyzoides & 3 & 4 & 150 & 23 & 1883 & 190 & -1.03 & 0.335 \\
\hline 26 & Gomphrena celosioides & 5 & 3 & 62 & 26 & 162 & 49 & -3.62 & 0.007 \\
\hline 27 & Panicum laxum & 6 & 4 & 74 & 209 & 39984 & 96 & -2.27 & 0.053 \\
\hline 28 & Aspilia Africana & 1 & 1 & 81 & 11 & 263 & 142 & -1.38 & 0.206 \\
\hline 29 & Ludwigia abyssinica & 4 & 3 & 69 & 24 & 602 & 101 & -1.81 & 0.108 \\
\hline 30 & Icacina trichantha & 1 & 2 & 148 & 17 & 98 & 60 & -3.36 & 0.011 \\
\hline 31 & Oplismenus burmannii & 41 & 33 & 82 & 105 & 3426 & 56 & -2.13 & 0.066 \\
\hline 32 & Paspalum conjugatum & 11 & 4 & 36 & 100 & 2553 & 50 & -3.96 & 0.004 \\
\hline 33 & Setaria barbata & 3 & 2 & 71 & 22 & 309 & 78 & -2.42 & 0.042 \\
\hline 34 & Phyllanthus amarus & 2 & 2 & 138 & 30 & 102 & 34 & -6.01 & $<0.001$ \\
\hline 35 & Sorghum arundinaceum & 1 & 1 & 224 & 53 & 129 & 21 & -10.3 & $<0.001$ \\
\hline 36 & Smilax anceps & 5 & 2 & 28 & 16 & 168 & 79 & -1.88 & 0.096 \\
\hline
\end{tabular}

systems (Rhoades, 1997). Some authors reveal that such influences may be necessitated by phytotoxins in the soil that may be plant-related (Harborne 1977, Rauha et al. 2000). Allelopathy is one of such plant-mediated influences that affect tree-plant interactions (Harborne 1977). Plants produce a large diversity of secondary metabolites including phenols and fatty acids which have an overall allelopathic effect on the growth and development of neighbouring plants species (Li et al., 2010). Other impeding factors may be poor availability of light necessitated by the tree canopy (Rauha et al. 2000). This means that those weeds or plants species that were located very close to the tree base would be sparsely abundant or distributed. This was the general observation about plant species abundance 
Table 9 Comparing diversity indices of plant species within or outside canopy demarcation of T. grandis

\begin{tabular}{llll}
\hline Parameters & Under canopy & Outside canopy demarcation & Remarks \\
\hline Taxa_S & 34 & 35 & Comparable taxa \\
Individuals & 1385 & 8577 & More individuals outside canopy demarcation (OC) \\
Dominance_D & 0.122 & 0.081 & More dominant spp. under canopy (UC) \\
Simpson_1-D & 0.879 & 0.920 & Comparable \\
Shannon_H & 2.656 & 2.954 & OC slightly higher sample diversity \\
Evennes__e^H/S & 0.419 & 0.548 & Species in OC more evenly distributed \\
Brillouin & 2.601 & 2.941 & OC slightly higher group diversity \\
Menhinick & 0.914 & 0.378 & UC with higher species richness than OC \\
Margalef & 4.562 & 3.754 & UC with higher species richness than OC \\
Equitability_J & 0.753 & 0.831 & Plants in OC probably more evenly distributed \\
Berger-Parker & 0.258 & 0.190 & The dominant spp. in UC is more abundant than that in OC \\
Chao-1 & 34 & 35 & Comparable taxa \\
\hline
\end{tabular}

*Calculation is based on the totality of weeds in all 5 quadrants

**The Chao-1 index is a measure of the abundance of individuals belonging to a certain class in a sample. In this case, we are looking at classes being individual species groups. The Berger-Parker index expresses the proportional abundance of the most abundant species

within $1.5 \mathrm{~m}$ from the base of the tree base; thus implying a negative single-tree influence of $T$. grandis on neighbouring plant diversity. It is, however, important to note that close pointy of the tree also enhanced the development of those plant species that were hitherto not found beyond the canopy.
In another development, the species abundance of some plants increased away from the canopy cover, whereas, for some, it decreased outside the cover than within the cover. Specifically, the growth of Sida garckeana, Reisantia indica, Momordica charantia and Tridax procumbens was completely impeded within $1.5 \mathrm{~m}$

Table 10 Bivariate correlation between soil physicochemical parameters and species abundance within and around tree canopy

\begin{tabular}{|c|c|c|c|c|}
\hline \multicolumn{5}{|l|}{ Correlations (Pearson's) } \\
\hline & Spa-UC (0-0.5 m) & Spa-UC (0.5-1.0 m) & Spa-UC (1.0-1.5 m) & Spa-OC \\
\hline $\mathrm{pH}(\mathrm{OC})$ & -0.486 & 0.315 & -0.422 & -0.176 \\
\hline Electric conductivity (OC) & 0.466 & 0.325 & 0.807 & 0.402 \\
\hline Total org. carbon (OC) & 0.249 & -0.379 & -0.658 & $-0.880^{*}$ \\
\hline Total nitrogen (OC) & -0.134 & -0.551 & 0.402 & 0.202 \\
\hline Potassium (OC) & -0.431 & 0.088 & -0.468 & 0.117 \\
\hline Calcium (OC) & -0.043 & 0.416 & 0.790 & $0.933^{*}$ \\
\hline Magnesium (OC) & -0.254 & 0.549 & -0.128 & 0.507 \\
\hline Soluble phosphates (OC) & -0.015 & -0.458 & -0.591 & $-0.906^{*}$ \\
\hline Sulphates (OC) & -0.259 & 0.315 & 0.675 & 0.856 \\
\hline $\mathrm{pH}(\mathrm{UC})$ & 0.454 & -0.572 & -0.330 & -0.675 \\
\hline Electric conductivity (UC) & -0.405 & 0.640 & 0.047 & 0.393 \\
\hline Total org. carbon (UC) & -0.174 & 0.482 & 0.710 & $0.916^{*}$ \\
\hline Total nitrogen (UC) & 0.045 & -0.404 & -0.568 & -0.400 \\
\hline Potassium (UC) & 0.534 & 0.154 & -0.484 & -0.391 \\
\hline Calcium (UC) & -0.340 & 0.457 & 0.520 & 0.857 \\
\hline Magnesium (UC) & -0.852 & 0.101 & -0.49 & -0.052 \\
\hline Soluble phosphates (UC) & -0.557 & 0.188 & 0.236 & 0.262 \\
\hline Sulphates (UC) & -0.078 & 0.521 & 0.800 & $0.884^{*}$ \\
\hline
\end{tabular}

*Correlation is significant at the 0.05 level (2-tailed)

A soil outside canopy demarcation, $U$ soil under canopy, Spa-UC species abundance under tree canopy, Spa-OC species abundance outside canopy demarcation 
from the tree. Being in close proximity to the base of the tree necessitated the development of Triumfetta cordifolia, which was not found in any other location other than under canopy within the forest. This might be due to the allopathic influence of $T$. grandis. Moreover, even though previous workers (Falk et al., 2008; Schnabel et al. 2017; Habashi and Waez-Mousavi, 2017) have reported a similar selective effect of single-tree on some plant species and soil microfauna, the mechanism is still unclear and may be short lived.

The possible association between soil physicochemical characteristics and plants species abundance under the single-tree influence suggested that increased sulphates in the soil might enhance plant species abundance under the influence of the tree canopy. Sulphates have been reported to enhance nutrient availability and acquisition by plants (Prade et al. 1993; Mitra et al. 2009). However, a negative association with phosphates was observed outside the tree canopy. Phosphorus is an essential macronutrient for plant growth, and it is limiting crop production in many regions of the world (Holford 1997). Increased phosphorus lead to increased plant development because phosphorus converts sunlight into usable energy, and essential to cellular growth and reproduction (Malhotra et al., 2018). The association statistics presented in Table 10 suggest both the negative and positive association between species abundance and total organic carbon under and outside canopies respectively. The negative association of this essential plant nutrient with plant species abundance within and beyond the canopy fringes calls for more scrutiny.

Species abundance outside the $1.5-\mathrm{m}$ radial demarcation positively correlated with the total organic carbon of soils in close proximity with the tree $(R=0.916, p<$ $0.05)$; the implication being that enhancing soil organic carbon with the tree canopy may be an important factor in increasing species abundance beyond this demarcated area. As reported earlier, total organic carbon within the $1.5-\mathrm{m}$ demarcated area to the tree was significantly higher than away from the area. Given the significant role organic carbon plays in plant species development, diversity and abundance through enhancing soil porosity, aggregate stability and water-holding capacity (Wehr et al. 2017), it is suggested that a reduction in organic matter of soil may have, amongst other biological and physicochemical factors, contributed to poor plant abundance of some plant species. Although there was generally more plant species outside the $1.5-\mathrm{m}$ demarcation than within, an increase in soil organic matter may further enhance such plant species abundance.

\section{Conclusion}

The single-tree influence of $T$. grandis on plant species abundance as well as characteristics of topsoil in an 8- year old planted forest has been investigated. Much as increased diversity of certain species was reported in close proximity to $T$. grandis, most of the plant species identified were negatively impacted very close to the tree. Given the fact that plant-plant associations affect the quality of forest soils, the impact of $T$. grandis in forest soil quality is possibly a factor of the outcome of its association with neighbouring plant species.

\section{Acknowledgements}

The researchers wish to thank the Management of the Moist Forest Research Station (Forestry Research Institute of Nigeria), Benin City, for providing space for the study.

\section{Funding}

Not applicable.

Availability of data and materials

Not applicable.

Authors' contributions

$\mathrm{BI}$ conceived the project idea and developed the research strategy with AL.

$\mathrm{AL}$ and $\mathrm{BI}$ collected the data while $\mathrm{MO}$ performed the statistical analysis. BI,

$\mathrm{Al}$ and $\mathrm{MO}$ contributed to writing the manuscript. All authors read and

approved the final version of the manuscript.

Ethics approval and consent to participate

Not applicable.

Consent for publication

Not applicable.

Competing interest

The authors declare that they have no competing interests.

\section{Author details}

'Department of Plant Biology and Biotechnology, Faculty of Life Sciences, University of Benin, Ugbowo, Benin City PMB 1154, Nigeria. ${ }^{2}$ School of Bioscience and Veterinary Medicine, University of Camerino - Center for Floristic Research of the Apennine, Gran Sasso and Monti della Laga National Park, San Colombo, 67021 Barisciano, L'Aquila, Italy. ${ }^{3}$ Moist Forest Research Station, Forest Research Institute of Nigeria, Benin City, Edo State, Nigeria.

Received: 13 January 2020 Accepted: 10 February 2020

Published online: 19 February 2020

\section{References}

Aborisade KD, Aweto AO (1990) Effects of exotic tree plantations of teak (Tectona grandis) and gmelina (Gmelina arborea) on a forest soil in south-western Nigeria. Soil Use and Management 6(1):43-45

Akindele SO (1989) Teak yields in the dry lowland rainforest area of. Nigeria Journal of Tropical Forest Science 2(1):32-36

Akobundu IO, Agyakwa CW. 1998. A Hand book of West African Weeds. International Institute of Tropical Agriculture, Ibadan 162 pp.

Aldrich JD. 1984. Weed-Crop Ecology: Principles and Practices, Breton Publishers, India. 1051-1060

Bray RH, Kurtz LT (1945a) Soil chemical analysis. Soil Science 59:39-45

Bray RH, Kurtz LT (1945b) Determination of total organic and available form of phosphorus in soils. Soil Science 59:45-49

Burgess SO, Adams MA, Turner NC, Ong CK (1998) The redistribution of soil water by tree roots systems. Oecologia 115:306-311

Cantarelli EB, Machado SLO, Costa EC, Pezzutti R (2006) Efeito do manejo de plantas daninhas no desenvolvimento inicial de Pinus taeda em várzeas na Argentina. Revista Árvore 30(5):711-718

Dinakaran J, Krishnayya NSR (2010) Variations in soil organic carbon and litter decomposition across different tropical vegetal covers. Current Sci 99(8):1051

Evangeline VR, Prakash EJJ, Samuel AS, Jayakumar M (2012) Allelopathic potential of Tectona grandis I on the germination and seedling growth of Vigna mungo. Pakistan Journal Weed Science Research 18(1):65-70

Falk K, Burke D, Elliott K, Holmes S (2008) Effects of single-tree and group selection harvesting on the diversity and abundance of spring forest herbs in deciduous forests in southwestern Ontario. Forest Ecology and Management 255(7):2486-2494. https://doi.org/10.1016/j.foreco.2008.01.033 
Habash H, Waez-Mousavi SM (2017) Single-tree selection system effects on forest soil microfauna biodiversity in mixed oriental beech stands. Applied Soil Ecology 123:441-446

Haluschak P. 2006. Laboratory Methods of Soil Analysis Canada-Manitoba Soil Survey. April 2006 P133p Available online: http://www.manitobaca/ agriculture/land/soil-survey/pubs/laboratory_methods_of_soil_analysispdf [Date assessed: 11/02/2016]

Harborne JB (1977) Introduction to Ecological Biochemistry. Academic Press, New York

Holford ICR (1997) Soil phosphorus: its measurement and its uptake by plants. Australian Journal of Soil Research 35:227-239

ICARDA. 2013. Methods of Soil Plant and Water Analysis: A Manual for the West Asia and North Africa Region Estefan G Sommer R and Ryan J (eds.). International Center for Agricultural Research in the Dry Areas. Box 114/5055 Beirut Lebanon 243p

Imoro ZA, Tom-Dery D, Kwadwo KA 2012. Assessment of soil quality improvement under Teak and Albizia Journal of Soil Science and Environmental Management 3(4): 91-96

Inderjit, Callaway RM (2003) Experimental designs for the study of allelopathy. Plant Soil 256(1):1-11

Izekor DN, Fuwape JA (2011) Performance of Teak (Tectona grandis L.F) wood on exposure to outdoor weather conditions. Journal of Applied Science and Environmental Management 15(1):217-222

Jia S, Wang X, Yuan Z, Lin F, Ye J, Hao Z, Luskin MS (2018) Global signal of topdown control of terrestrial plant communities by herbivores. Proceedings of the National Academy of Sciences of the United States of America 115(24): 6237-6242. https://doi.org/10.1073/pnas.1707984115

Jose S, Allen SC, Nair PKR. 2008. Tree-crop interactions: lessons from temperate alley-cropping systems. In : DR Batish RK, Jose KS, Singh HP (eds.). Ecological Basis of Agroforestry. CRC Press, Boca Raton Fl. pp 15 - 36.

Kanazawa KK, Higuchi NK, Nishizawa S, Fushiya M, Chino M, Mori M (1994) Nicotianamine aminotransferase activities are correlated to the phytosiderophore secretion under Fe-deficient conditions in Gramineae. J Exp Bot 45:1903-1906

Kole RK, Karmakar PR, Poi R, Mazumdar D (2011) Allelopathic inhibition of teak leaf extract: A potential pre-emergent herbicide. Journal of Crop and Weed 7(1):101-109

Lal R (2005) Forest soils and carbon sequestration. For Ecol Manag 220:242-258 Leopold AC, Salazar J (2008) Understory species richness during restoration of wet tropical forest in Costa Rica. Ecological Restoration 26(1):22-26

Li ZH, Wang Q, Ruan X, Pan CD, Jiang DA. 2010. Phenolics and plant allelopathy. Molecules (Basel, Switzerland). 15(12): 8933-8952. doi:https://doi.org/10.3390/ molecules15128933

López-Pintor A, Espigares T, Rey-Benayas JM, Gómez-Sal A (2000) Effect of simulated parent-created microenvironmental conditions on germination of Retama sphaerocarpa (L) Boiss seeds. Journal of Mediterranean Ecology 1:219-226

Malhotra H, Sharma VS, Pandey R (2018) Phosphorus nutrition: plant growth in response to deficiency and excess. In: Fujita M, Oku H, Nahar K, HawrtlakNowak B (eds) Hasanuzzaman, M. Plant Nutrients and Abiotic Stress Tolerance, -171, 190 10.1007\%2F978-981-10-9044-8_7

Manimegalai AA, Manikandan TB, Sheela RC, Geetha S (2013) Allelopathic influence of Tectona grandis leaves on the germination of black gram and green gram. International Journal of Curriculum Science 1:241-244

Maraschin-Silva F, Aqüila MEA (2006) Potencial alelopático de espécies nativas na germinação e crescimento inicial de Lactuca sativa $L$ (Asteraceae). Acta Botanica Brasilica 20(1):61-69

Merino-Gergichevich C, Alberdi M, Ivanov AG, Reyes-Diaz M (2010) $\mathrm{Al}^{3+}-\mathrm{Ca}^{2+}$ interaction in plants growing in acid soils: Al-phytotoxicity response to calcareous amendments. Journal of Soil Science and Plant Nutrition 10(3):217-243

Mitra GN, Sahu SK, Nayak RK. 2009. ameliorating effects of potassium on iron toxicity in soils of Orissa Presentation at the IPI-OUAT-IPNI International Symposium 5-7 November 2009 OUAT Bhubaneswar Orissa India

Nasir R, Khan M, Masab M, Rehman HU, Rauf NU, Shahab S, Ameer N, Sajed M, Ullah M, Fafeeq M, Shaheen Z. 2015. Accumulation of Heavy Metals (Ni Cu Cd Cr Pb Zn Fe. in the soil water and plants and analysis of physico-chemical parameters of soil and water Collected from Tanda Dam Kohat. J Pharm Sci \& Res 7(3):. 89-97

Ong CK, Deans JD, Wilson JM (1999) Exploring belowground complementarity in agroforestry using sap flow and root fractal techniques. Agroforestry Systems 44:87-103

Oyebade BA, Anaba JC. 2018. Individual tree basal area equation for a young Tectona grandis (Teak. plantation in Choba Port Harcourt Rivers State Nigeria. World News of Natural Sciences 16:144-154
Pellet MD, Grunes DL, Kochian LV (1995) Organic acid exudation as an aluminium tolerance mechanism in maize (Zea mays L.). Planta 196:788-795

Prade K, Ottow JCG, Jacq VA. 1993. Excessive iron uptake (iron toxicity. by wetland rice (Oryza sativa L.) on an acid sulphate soil in the Casamance /Senegal International Institute for Land Reclamation and Improvement Wageningen The Netherlands ILRI Publication 44: 150-162

Rao MR, Nair PKK, Ong CK (1998) Biological interactions in tropical agroforestry systems. Agroforestry Systems 38:3-50

Rauha JS, Remes M, Heinonen A, Hopia M, Kahkonen T, Kujala K, Pihlaja H, Vuorela P (2000) Antimicrobial effects of Finish plant extracts containing flavonoids and other phenolic compounds. International Journal of Food Microbiology 56:507-517

Rhoades CC (1997) Single-tree influence on soil properties in agroforestry: Lessons from natural forest and savannah ecosystem. Agroforestry Systems 35 $71-94$

Riginos C (2009) Grass competition suppresses savannah tree growth across multiple demographic stages. Ecology 90(2):335-340

Salt DE, Prince RC, Baker AJM, Raskin I, Pickering IJ (1999) Zinc ligands in the metal hyperaccumulator Thlaspi caerulescens as determined using X-ray absorption spectroscopy. Environ Sci Technol 33:713-717

Schnabel F, Donoso PJ, Winter C (2017) Short-term effects of single-tree selection cutting on stand structure and tree species composition in Valdivian rainforests of Chile. New Zealand Journal of Forestry Science 47:21. https://doi. org/10.1186/s40490-017-0103-5

Scrivanti LR, Zunnino MP, Zygadlo JA (2003) Tagetes minuta and Schinus areira essential oils as allelopathic agents. Biochemical Systematic and Ecology 31(6): 563-572

Silva PSL, Silva PIB, Silva KMB, Oliveira OF, Jales JDD, Medeiros JLB (2010) Weed community and growth under the canopy of trees adapted to the Brazilian semi-arid region. Planta Daninha Viçosa-MG 28(1):69-76

SSSA. 1971. Instrumental Methods for Analysis of Soil and Plant Tissue. Soil Science Society of America Corporated Wisconsin USA Pp27-32

Thakur M, Eisenhauer N (2015) Plant community composition determines the strength of top-down control in a soil food web motif. Scientific Reports 5 : 9134. https://doi.org/10.1038/srep09134

Watanabe Y, Masunaga T, Owusu-Sekyere E, Buri MM, Oladele OI, Toshiyuki WT (2009) Evaluation of growth and carbon storage as influenced by soil chemical properties and moisture on teak (Tectona grandis) in Ashanti region. Ghana J Food Agric Environ 7(2):640-645

Wehr JB, Smith TE, Menzies NW (2017) Influence of soil characteristics on teak (Tectona grandis $L$ f.) establishment and early growth in tropical Northern Australia. Journal of Forest Research 22(3):153-159

Zinke PJ (1962) The pattern of influence of individual forest trees on soil properties. Ecology 43(1):130-133

\section{Publisher's Note}

Springer Nature remains neutral with regard to jurisdictional claims in published maps and institutional affiliations.

\section{Submit your manuscript to a SpringerOpen ${ }^{\circ}$ journal and benefit from:}

- Convenient online submission

Rigorous peer review

- Open access: articles freely available online

High visibility within the field

- Retaining the copyright to your article

Submit your next manuscript at $\boldsymbol{\nabla}$ springeropen.com 